\title{
Considerazioni biologiche sui voli spaziali eseguiti fino ad oggi (*)
}

\author{
T. Lomonico $(* *)$
}

Ricevuto il 19 Febbraio 1963

Riassunto. - L'A. espone dapprima i risultati di interesse biologico conseguiti nei primi voli spaziali, suborbitali ed orbitali, realizzati dai sovietici, dagli americani e dai francesi, con l'impiego di animali da esperimento di specie diverse. Sul presupposto di tali ricerche venivano lanciati, per voli orbitali, i sovietici Gagarin e Titov.

Nei due astronauti le modificazioni delle grandezze circolatorie durante la fase di ipergravita non differivano dai dati rilevati in centrifuga. In assenza di gravita le variazioni cardiache e respiratorie furono quasi nulle, mentre non si rilevarono disturbi nei movimenti e nella coordinazione muscolare.

Solo 'Titov, per qualche istante del volo, presentò vertigrini e nausea che insorgevano con i movimenti della testa.

Gli Stati Uniti eseguivano i loro prini voli spaziali umani suborbitali, con i lanci di Shepard e Grissom.

Dopo aleuni voli orbitali sperimentali realizzavano il loro primo volo orbitale umano con il lancio del tenente colonnello Glenn. I dati biologici teletrasmessi fecero rilevare un comportamento dell'apparato cardiovascolare, nelle fasi di iper e di zero gravita, che rientrava nelle previsioni. Le funzioni sensoriali non presentarono alterazioni, ove si eccettui una riduzione della visione crepuscolare, né si ebbero, in condizioni di zero-gravità, disturbi labirintici, che pure l'astronauta cercò di provocare con movimenti della testa. L'alimentazione, eseguita con cibi appositamente preparati, non presentò difficoltà.

Il 24 Magrgio 1962 veniva lanciato, per un volo orbitale, l'americano Carpenter. Questi presentò, in condizioni di subgravità, tachicardia e notevoli variazioni nella pressione arteriosa.

L'A. conclude la rassegna dei dati biologici raceolti nel corso dei voli spaziali eseguiti da uomini e da animali da esperimento, facento rilevare co-

$\left(^{*}\right)$ Nota presentata al $2^{\text {o }}$ Congresso Internazionale Tecnico Scientifico dello Spazio. Roma, 19-23 Giugno 1962.

(**) Ispettorato di Sanità Aeronautica. Capo dell Ispettorato: T'en. Gen. med. C.S.A. Prof. Dott. T. Lononaco. 
me si siano ottenuti dati piuttosto contrastanti sulle condizioni cardiovascolari e labirintiche in zero-gravità che richiederanno nuove indagini nel corso di ulteriori voli spaziali.

Sunnary. - The Author exposes the results of biological interest obtained through the first space flights (suborbital or orbital) executed by soviet, American, and French scientists, employing different animals. On the basis of such scientific acquisitions, the Soviet astronauts Gagarin and Titov were launched into orbital flights.

Both of them did not underwent, in the active phase of the flight, any special change of cardiocirculatory or respiratory functions, which was not previously observed on the ground in oceasion of centrifuge tests. During weightlessness any change was practically absent, and no troubles of mobility or muscolar coordination were recorded.

Titov only, for a period of a few moments, suffered from nausea and dizziness, provoked by head movements.

The first U.S.A. suborbital flights were accomplished by Slepard and Grissom.

After some experimental orbital flights, the U.S.A. executed their first manned orbital flight. The telemetration of the biologieal parameters concerning Jt. Col. Glenn showed that the behaviour of the cardiovascular apparatus in hyper-and zero-gravity corresponded to the previsions. The sensory functions did not underwent any special clange, except for a reduction of twilight vision. Furthermore 10 labyrinthine disturbances were observed in zero-gravity, although the astronant attempted to elicit them by means of voluntary head movements. Feeding with especially packed food did not offer particular difficulties.

On May 24, 1962, the American pilot Carpenter was put into orbit. During weightlessness he presented tachycardia and very large changes in blood pressure.

At the end of lis survey of the biological data recorded during space flights of men and animals, the Author points out that the so far collected data are still scarcely homogeneous, as far as cardiovascular and labyrinthine conditions in zero-gravity are concerned, so that a large amount of investigations, to be conducted in future space fliglits, is still required.

Ia serie ininterrotta di voli suborbitali ed orbitali eseguiti fino ad oggi nonchè le ricerche scientifiche effettuate al riguardo con varie modalità e con vari mezzi di laboratorio ei obbligano a fare il punto della ricerea bio-spaziale onde consentire, se possibile, un bilancio.

Da questo si potrebbero trare considerazioni ed argonentazioni per un migliore indirizzo delle future ricerche mediche aerospaziali.

Ecro qui una elencazione descrittiva dei voli spaziali biologici eseguiti fino ad ogeri. 
Periodo che va dal 1949 al 1952.

J. P. Henry e Coll., ricercatori statunitensi, sperimentarono in roli con V2 ed Aerobee il comportamento del labirinto di alcuni animali in condizioni di subgravità. Gli animali usati in queste ricerche furono i topini, alcuni con labirinto integro ed altri slabirintati. Fu registrato a mezzo films e con fotografie il comportamento motorio di questi animali. Fu constatato che nel periodo di galleggiamento provocato della subgravità i topini slabirintati presentavano un migliore orientamento rispetto a quelli forniti di labirinto integro.

1957 .

Durante l'anno 1957 il russo A. M. Galkin e collaboratori lanciarono a più riprese coppie di cani (uno anestetizzato ed uno normale) ad una quota di circa $200 \mathrm{~km}$ raggiungendo una assenza di peso della durata di 3,5 minuti. I cani venivano piazzati in un contenitore stagno racchiuso nell'ogiva di un razzo. In questo contenitore si aveva una pressione atmosferica che variava da 760 a $460 \mathrm{~mm}$ di $\mathrm{Hg}$ : la concentrazione di $\mathrm{CO}_{2}$ veniva mantenuta quasi normale con assorbitori e $\mathrm{l}^{\prime} \mathrm{O}_{2}$ consumato veniva reintegrato automaticamente.

Durante il lancio venivano telemetrati: l'ecg, il respiro, la pressione arteriosa.

La registrazione di questi dati mostrò un aumento massimo durante le fasi "attive » ciò durante il lancio e durante il rientro in atmosfera; durante lo zero-gravità tutti i valori fisiologici tornavano normali, ma non così rapidamente come era da aspettarsi.

Negli animali anestetizzati le variazioni fisiologiche durante le fasi "attive" furono meno pronunziate.

Nello stesso anno apparvero le osservazioni da parte dei russi V. N. Chernor e V. Y. Yakolev sul comportamento della cagnetta "Laika " in volo orbitale.

Il 3 Novembre 1955 daggli scienziati russi fu lanciato e messo in orbita circum-terrestre un satellite contenente in un speciale abitacolo la cagnetta "Laika ".

Prima del lancio della cagnetta fu eseguita una accurata preparazione biologica di alcuni cani dai quali doveva poi essere scelta "Laika " del peso di $\mathrm{kg} 6$ circa. La preparazione aveva il fine di conoscere per ogni animale i dati medi delle loro variabili fisiologiche e di ottenere un adeguato addestramento in maniera che l'animale prescelto non presentasse 
reazioni enotive esagerate durante le fasi critiche del volo orbitale e sopportasse senza danni per parecehio tempo un ambiente piccolo e confinato, qual'ela appunto quello della capsula spaziale, a forma oblunga con diametro di 64 cm e lunghezza di $80 \mathrm{~cm}$.

A tal fine gli animali furono per parechie volte esaminati in centrifuga e si ottenne da essi l'abitudine a stare rinchiusi in uno stretto abitarolo con la diminuzione progressiva del volume dello abitacolo stesso: inoltre gli animali furono abituati, attraverso l'instaurarsi di riflessi condizionati, ad alimentarsi e ad evacuare ad ore prestabilite.

L'animale prescelto fu posto su un comodo supporto e legato con (inghie che però gli permetterano di stare all'impiedi, di sedere, di chinarsi ed anche di muoversi un po' avanti ed indietro.

La capsula conteneva una sostanza assorbente $\mathrm{CO}_{2}$ e rigeneratrice di $\mathrm{O}_{2}$. L'alimento consisteva in una miscela gelatinosa di acqua, di erosta di pane, di earne polverizzata e di grasso di manzo. La consistenza gelatinosa aveva il fine di ottenere un maggiore attrito alle pareti del recipiente e quindi mitigare la galleggiabilità durante la subgravità.

Vi era poi un dispositivo per accogliere gli escrementi e le urine.

Le grandezze fisiologiche venivano telemetrate. Esse furono: ecg, frequenza respiratoria, pressione arteriosa, comportamento motorio. Chernov e Yakolev riferiscono che tutte queste grandezze rimasero normali in tutte le fasi del volo, eccetto durante la fase iniziale, ciò̀ dumnte le accelerazioni in cui vi fu un aumento che però si presento sempre meno mareato pur aumentando i valori di accelerazione.

Raggiunto il periodo di subglavità tutto tornò alla norma ma dopo un periodo di tempo più lungo di quello medio riscontrato durante le prove ellettuate a terra dopo le aceelerazioni in centrifuga.

1958.

Nel 1958 B. G. Bugrov dell'U.R.S.S. e collaboratori eseguirono esperimenti per accertarsi se fosse stata possibile la vita di cani lacechiusi in indumenti a pressione con casco trasparente.

A tale scopo furono lanciate capsule fino a raggiungere quote di circa $110 \mathrm{~km}$. Dopo una breve traiettoria ed a quota più bassa i cani venivano lanciati fuori della capsula con paracadute. Vi furono parecchie perdite di animali, ma alcuni raggiunsero in buone condizioni la superficie terrestre. Dumante il volo furono come al solito registrati i vari dati fisiologici che presentarono variazioni simili a quelli degli esperimenti di Galkin. 
Nello stesso anno gli Statunitensi F. L. Van der Wal e W. D. Young lanciarono come da progetto "Mia ", topini in roli orbitali con missili Thor-Able dumante i quali si ebbero periodi di subgravità di durata notevole $\left(45^{\prime}\right)$. I dati fisiologici ricavati però non furono molto conclusivi.

Semple nel 1958 si effettuò il "Joint Army Navy Bioflighlt Project 1 ". Venne lanciata una scimmia (la scimmia "Gordo" entro una ogiva di un Jupiter IRBM.

L'animale non fu recuperato. Durante il lancio furono teletrasmessi i seguenti dati biologici: eeg, frequenza respiratoria, toni cardiaci, temperatura cutanea, temperatura intema della capsula. Son tutti questi dati furono però trasmessi chiari.

Comunque fu riscontrato come al solito un ammento della frequenza cardiala durante il lancio con ritomo parziale ai dati normali durante la fase di gravità zero.

1960.

Tel 1960 ebbe luggo l'esperimento russo con le eagnette Bella e Strelka, ambedue recuperate.

I1 19 Agrosto 1960 l'Unione Sorietica lanciò una secondia navo spaziale attorno alla terra del peso di $4600 \mathrm{~kg}$.

La nave era divisa in 2 scomparti: cabina per gli animali e cabina per gli strumenti.

Lo scomparto biologico conteneva due cagnette "Belka e Strelka " pesanti rispettivamente $4,5 \mathrm{~kg}$ e $5,5 \mathrm{~kg}$ e gabbie con ratti e topini albini e neri, centinaia di insetti, semi di cipolla, piselli, orzo, mais eec. alghe del tipo Chlorella, bacilli intestinali, fagri, cellule epiteliomatose umane, pelle umana e di coniglio ece.

I cani furono condizionati nella maniera simile a quella usata per Laika. In essi fu esteriorizzata la carotide per eseguire misure di pressione arteriosa e furono inseriti sottorute due elettrodi per la trasmissione dei dati pressori sanguigni ed elettrocardiografici.

O!tre agli strumenti per la telemetria dei principali eventi fisiologici dentro la capsula fu allogata anche la televisione.

Le condizioni nell'interno dell'abitacolo elano le seguenti: P.B. uguale a quella del livello del mare, $\mathrm{CO}_{2}$ inferiore all'1\%, temepratura $15-25$ of., umidità relativa $30-70 \%$.

$\mathrm{I}$ : O consumato, il $\mathrm{CO}_{2}$ espirato e l'acqua emessa renivano adeguatamente regolati con corrispondente emissione di $\mathrm{O}_{2}$, assorbimento di $\mathrm{CO}$, 
e $\mathrm{H}_{2}$ O. La regolazione eja automatica ma comunque ogni variazione (era telemetrata. L'aumento interno della temperatura reniva anch'essa tenuta costante a mezzo di refrigeratore radiante ad aria liquirla.

Per la alimentazione fu fatto uso, come nel caso di Taika, di sostanze gelatinose ricehe di alimenti nutritivi ed acqua.

Ia sostanza gelatinosa arleriva, come si è detto, ai recipienti e quindi non si stacoava dal fondo dei recipienti stessi in subgravità. L'alimento era messo a disposizione degli animali a intervalli fissi, tramite l'apertura automatica di un rompartimento.

Al $18^{\circ}$ giro orbitale la nave fu fatta scendere.

Essa fu immessa in una traiettoria discendente; l'abitacolo, separato dal resto, vemne paracadutato a $7 \div 8.000 \mathrm{~m}$ e durante l'apertura del congegno ritardante si raggiunse una decelerazione di circa $10 \mathrm{G}$.

I dati trasmessi dumante il volo orbitale sembra che abbiano dimostrato che tutto si sia comportato normalmente.

All'arrivo le cagnette ed i topini non presentavano segni di sofferenzal.

I)egli studi ulteriori proseguiti sul notevole materiale biologico trasportato dat satellite nel volo orbitale nulla ancora si sa anche perehè è evidente che per i definitivi risultati dovrà passare molto tempo sopratutto se si tiene conto degli effetti proiettati nel futuro delle lesioni genetiche provocate dalle maliazioni ionizzanti.

Apparirà subito l'importanza rli questo notevole esperimento russo quando si sa che furono anche trasportati nel rolo orbitale culture di particolari batteri i quali sotto l'azione delle radiazioni ionizzanti si potevano mutare in altri tipi di batteri ard es. i batteri lisogenici che producono i batteriofogi). Furono anche messi in orbita tubi da saggio contenenti cellule cancerigne per studiatre dopo il volo le eventuali modifirazioni ed ampolle contenenti ac. desossiribonucleico.

Informazioni conclusive e nello stesso tempo più particolareggiate sugli eventi fisiologicei in volo spaziale ci vengono date da Gazenko e Yazdovsky (1961). Questi autori di dicono che in alemi degli animali lanciati nello spazio furono registrati, dumente le prime ore di assenza di peso, cambiamenti di intensità dei rumori del cuore. Il 10 tono infatti si presentava più prolungato la qual cosa starebbe per un aumento della durata di espulsione del ventricolo sinistro. Questo fenomeno scompare più o meno rapidamente, ma dopo $8 \div 10$ ore appaiono altre modificazioni.

Si nota infatti un aumento della frequenza del polso, della irequenza del respiro e della pressione arteriosa. Anche l'onda T dellecer appare deformata. 
Si deve poi sottolineare un altro fenomeno: la difficolta in regime zero-gravitazionale del ritorno ai valori normali circolatori dopo le variazioni presentate a causa delle accelerazioni allatto della partenza.

Questo fenomeno fu chiaramente osservato sulla cagnetta Laika nella quale durante il periodo di imponderabilità il tempo di ritorno alla norma delle grandezze circolatorie fu 3 volte più lungo che in condizioni di gravità normale ed anzi qualche grandezza come la frequenza cardiaca si mantenne per un certo tempo sotto i valori normali.

1961.

Nel 1961 si compi l'esperimento statunitense di rolo suborbitale con un razzo vettore Redstone contenente una capsula con lo scimpanzè " IIam ".

Il 31 Gemnaio 1961 da Cape Camaveral fu lanciato come è noto, uno scimpanzè, posto in una capsula simile a quella dell'attuale progetto Mereury. L'altezza massima raggiunta fu $313 \mathrm{~km}$ e la gittata $675 \mathrm{~km}$. La capsula cardde a 175 miglia ad ovest dell'isola Grand Bahama e fu recuperata con lo scimpanzè vivo.

Iurante il volo furono teletrasmessi vari dati fisiologici e fra questi anche le reazioni motorie dell'animale.

Queste ultime furono riscontrate normali durante il volo: lanimale recuperato non presento segni di sofferenza o disfunzioni di rilievo.

Il 9 Marzo 1961 da località ancola sconosciuta fu lanciato dai russi in volo orbitale lo Sputnik IX (korabl III) rontenente la cagnetta Cernuska ed altri oggetti biologici non meglio dichiauati.

Lo Sputnik compi 2 giri e un atterraggio, nessuna lesione fu riscontrata alla cagnetta.

Questo volo orbitale deve essere considerato come preparatorio al prossimo volo orbitale umano compiuto da parte pussa.

Il 23 Marzo 1961 vemne ancora dai russi lanciato in volo orbitale lo Sputnik X (kolabl IV) con la cagnetta Svezdoscia.

I dati fisiologici di questa cagnetta telemetrati durante il rolo ripeterono i dati ottenuti con la cagnetta Cermuska. Questo rolo renne adempinto dai russi nell'intento di ottenere ulteriore sicurezza per il prossimo lancio umano.

Ed infatti il 12 Aprile 1961, alle ore 9,07 (ora di MLosca) un missile tre-quadristadio, il Vostok Io venne lanciato dai russi da località ancora non conosciuta. Il missile portava una cabina contenente, per la prima volta nella storia dell'umanità, un uomo: il maggiore ventisettenne Guri Alexeievich Gagarin. 
Il peso della cabina era di $4725 \mathrm{~kg}$. Laltezza del perigeo fu di $181 \mathrm{~km}$, quella dell'apogeo $327 \mathrm{~km}$.

Gagarin rimase in volo $108^{\prime}$ di cui $80^{\prime}$ di viaggio vero e proprio nello spazio e quindi in fase di gravità zero. Bggli circummavigì $\tilde{5}$ volte il pianceta terrestre.

Il 6 Agosto 1961 un altro russo remme lanciato in rolo orbitale con i) Vostok II da una base anche questa non ancora conosciuta del territorio sovietico. I il maggiore Gherman Stefanovic Titov di 26 anni.

La capsula contenente il cosmonanta partiva alle ore 7 (ora di Mosca) - rimaneva in orbita $25^{\text {h }}$ e $18^{\prime}$. Ijatterraggrio avveniva alle ore 8,18 del 7 Agosto presso Samatov (Basso Volga). Il peso della capsula era di $4731 \mathrm{~kg}$ : l'apogeo $257 \mathrm{~km}$, il perigeo $178 \mathrm{~km}$. Titov compì 17 circummavigazioni attorno il pianeta terrestre.

Prima di dire qualche cosa sugli eventi fisiologici registrati da questi cosmonauti russi è bene spendere una parola sulla loro selezione fisiologica e sul loro addestramento.

In questi cosmonauti si sono pretesi imnanzi tutto le più alte qualità professionali: dal punto di vista medico nessuna deviazione delle funzioni fisiologiche e psicologiche; costituzione fisica ben sviluppata; un grande grado di stabilità emozionale. Nell'esame di selezione oltre i metodi classici furono impiegati la camera a depressione, la centrifuga, strumenti viblanti a strumentario misurante il grado di stabilità labirintica.

Iungamente poi i piloti furono addestrati in centrifuga, in camera a depressione e mediante dispositivi strumentali abituati a disquilibri funzionali vestibolari.

Sia in Gagarin che in Titov durante la fase attiva dei voli i rati telemetrati per quanto riguarda la funzione circolatoria e respiratoria furono simili a quelli riscontrati durante le prove a terra in centrifuga. In Titov la frequenza cardiaca raggiunse qualche volta punte più elevate $\left(118 \div 134\right.$ al $\left.1^{\prime}\right)$.

Durante la fase di imponderabilità le variazioni cardiache e respiratorie furono quasi nulle.

Durante il sonno (sempre in condizioni di imponderabilita) la frequenza cardiaca di Titor adde da $80 \div 100$ per $1^{\prime}$ a $84 \div 58$ per $1^{\prime}$.

Durante il periodo di imponderabilità i due astronauti hamno conservato ogni loro capacità e la possibilità di ogni movimento complicato: la coordinazione muscolare fu sempre ottima.

Per qualche istante del volo Titov risentì aleuni disturbi subiettivi di origine vestibolare: un leggero stato di vertigine e nausea specialmente quando egli roltava la testa o quando si spostavano rapidamente 
oggetti davanti ai suoi occhi. Questi disturbi non limitarono la capacità mentale o muscolare del cosmonauta.

Intanto anche in America si incomincia a pensare l'invio nello spazio di qualche cosmonauta.

Il 28 Aprile 1961 venne fatta una prova generale per il recupero di una capsula in mare. Questa venne infatti lanciata con il rettore Little Jol $V$ e l'esperimento riusci bene.

Il 5 Maggio 1961 alle ore 9,34 da Cape Canaveral il capitano di Marina Alan B. Shepard iniziava il primo rolo suborbitale statunitense (Mercury Freedom 7). Alle ore 9, 49 la capsula cadeva in mare vicino alla portaerei "Lake Champlain ". Missile vottore fu un "Redstone ": altezza raggiunta $136 \mathrm{~km}$; gittata $483 \mathrm{~km}$.

Durante questo volo lo Shepard fu sottoposto nella fase di lancio a circa 8 G di accelerazione e quindi entrò in gravità-zero per circa $5^{\prime}$ : nel rientro in atmosfera fu sottoposto a circa $10 \mathrm{G}$ di decelerazione.

I dati fisiologici presi accuratamente prima, durante e dopo il rolo sono stati ampiamente pubblicati.

Da essi appare quanto segue:

1. L'astronauta fu durante il volo in completa "performance " psico-fisica.

2. Durante i $5^{\prime}$ di assenza di gravità non si verificò nessun evento fisio-patologico di rilievo.

3. Durante la transazione del periodo di accelerazione al periodo di imponderabilità i dati delle risposte fisiologiche caddero entro i limiti della normalità.

La frequenza del polso durante il passaggio dello zero-gravità al periodo di rientro in atmosfera si presentò uguale a quella che prima più volte si era osservata quando il pilota in centrifuga umana passava da $1 \mathrm{G}$ a più $\mathrm{G}$.

4. Le funzioni degli organi dei sensi, specialmente visione, organi restibolari ed udito rimasero intatti durante il volo.

Il secondo lancio suborbitale americuno, Mercury Liberty Bell 7 , ̀̀ arvenuto il 21 Luglio 1961 da Capo Canaveral alle ore 7,20 con a bordo il Capitano U.S.A.F. Virgil T. Grissom (detto Guss).

Il rolo si compì 16' dopo con conclusione drammatica. Lo sportello di sicurezza detla capsula si apriva per cause imprecisate e la capsula piena d'acqua affondava sebbene agganeiata da un elicottero della portaerei "Randolph ". L'astronauta veniva salvato. Il missile vettore fu il "Rerlstone 4 "; l'altezza massima ragoiunta $189 \mathrm{~km}$, la gittata $488 \mathrm{~km}$. 
Nel rolo suborbitale di Grissom si compirono gli stessi eventi fisiologici di quelli registrati durante il volo di Shepard. Ovviamente parecehi dati registrati andarono perduti a causa dell'affondamento della capsula.

Il 13 Sottembre 1961 fu lanciato da Cape Canaveral (Nereury IV) un "crewman - simulator", cior un "robot " con un missile Atlas.

Questo rimase in orbita 89' c cadde vicino alle Bermule.

Fu un volo preparatorio a quello umano e con esso si volle vedere se la capsula che doveva in seguito portare in orbita il futuro cosmonauta fosse resistita alle aceelerazioni ed alle decelerazioni.

L'esperimento riusci oftimamente.

Il 29 Settembre fu eseguito un 20 esperimento preparatorio "il Mereury $\mathrm{V}$ \%.

Ia Cape Canaveral fu messa in orbita una capsula uguale a quella che sarebbe servita per il volo umano: dentro questa capsula fu collocato lo scimpanzè "Enos" di cinque amni, del peso di $\mathrm{kg} 17$ ed alto $96 \mathrm{~cm}$.

Il missile vettore fu un Atlas. La capsula raggiunse un apogeo di $236 \mathrm{~km}$ exl un perigeo di 120.

Questa partita alle ore 10,07 compiva solo 2 dei 3 giri prestabiliti a causa dell'intenso calore sviluppatosi.

Il lancio non riusciva a perfezione.

Il 20 Febbraio 1962 gli Stati Uniti lanciarono da Cape Canaveral il primo astronauta nello spazio: il Ten. Col. dei marines John Glenn ron il "Friendship Seven ".

La durata del volo fu di $4^{\text {h }}$ e $56^{\text {t }}$ con $4^{\text {h }}$ e $2 \tau^{\prime}$ di imponderabilità.

Il lazzo vettore fu un "Atlas". L'apogeo fu di $261 \mathrm{~km}$, il perigeo di $157 \mathrm{~km}$.

Ecco una sommaria descrizione dei vari eventi fisiologici presentati dal cosmonauta durante l'impresa spaziale.

Il pilota entrò verso le 6 del mat tino nella capsula spaziale. Indossava la tuta spaziale. Si distese sul materassino sagomato in posizione supina con la testa e le spalle alzate dal piano orizzontale di 120 e le anche ed $\mathrm{i}$ ginocchi flessi a $90^{\circ}$. Cinture attorno alle spalle e all'addome assicuravano il suo corpo al materassino.

Dopo il lancio a causa della inclinazione della capsula egli si trovò non più in posizione supina ma seduto con le spalle in direzione del moto. Durante il rientro per il nuovo assetto preso dalla capsula egli si trovò nuovamente in posizione supina sempre con le spalle in direzione del moto. Dentro la capsula egli era mantenuto in una atmosfera di $\mathrm{O}_{2}$ al 100/100. Il tempo totale trascorso dentro la capsula prima del lancio fu di 3 ore e $41^{\prime}$. La partenza si srolse secondo il programma. 
Le accelerazioni raggiunsero valore di $6,7 \mathrm{G}$ dopo $10^{\prime \prime}$ dal tempo di partenza ciò̀ nel tempuscolo che immediatamente precede il distacco del $1^{\circ}$ stadio del missile (BECO) dopo di che le accelerazioni raggiunsero un valore di $1,4 \mathrm{G}$. Esse raggiunsero in $2^{\prime}$ e $52^{\prime \prime} 7,7 \mathrm{G}$ nel tempuscolo immediatamente antecedente al distacco del 20 stadio (SECO). Indi iniziò il periodo di assenza di gravità che si protrasse $4^{\text {th }}$ e $38^{\prime}$. Al rientro si ebbe una nuova punta aceelerativa di $3,7 \mathrm{G}$ all'atto dell'apertura del paracadute principale.

I dati fisiologici principali teletrasmessi riguardavano: ecg con due derivazioni, frequenza respiratoria, temperatura corporea, sfigmometria.

Questi dati venivano rilevati da appositi biosensori e i tracciati raccolti a terra furono sempre buoni.

La frequenza cardiaca al momento della partenza ed al momento della separazione del missile vettore dalla nave spaziale raggiunse 114 battiti al 1'. Durante il periodo dell'imponderabilità la frequenza cardiaca variò da 88 a 114 battiti al 1' nei primi $10^{\prime}$ e nel restante periodo di zerogravità si mantenne ad una media di 86 battiti al $1^{\prime}$.

Nel rientro il polso si mantenne a 96 , tranne al momento della apertura del paracadute nel quale raggiunse i 134 .

L'ecg mostrò ritmo sinusale normale con qualche aritmia.

I valori di pressione arteriosa variavano da 119 a 143 mm $\mathrm{Ho}$ per la Mx e da 60 a 81 per la Mn.

È da tenere presente che nella mattinata del lancio i valori riscontrati furono i seguenti: frequenza cardiaca 68, pressione 118/80.

Il pilota esegui anche un certo lavoro muscolare per breve periodo, consistente in movimenti degli arti superiori, di estensione e di flessione nell'atto di stirare i cavi elastici di un attrezzo ginnico (ergometro).

L'esercizio fu eseguito sopra Zanzibar durante la $1^{\mathrm{a}}$ orbita. La frequenza cardiaca salì durante questo lavoro muscolare da 80 a 120 al $1^{\prime}$ in $30^{\prime \prime}$. Dopo $2^{\prime}$ dell'esercizio, però il polso ritornò a 84 .

Per la temperatura corporea si constato un leggero aumento progressivo da $36,5^{\circ} \mathrm{C}$ a $37,5^{\circ} \mathrm{C}$, calusato da un non perfetto condizionamento dell'atmosfera interna della capsula.

Le commicazioni fatte a voce dall'astronanta durante il volo erano accurate e coerenti durante tutto il periodo del volo. La voce per se stessa dava la sensazione di benessere e lo stato mentale appariva appropriato alla situazione. Anche l'umore ela normale. Le pronte risposte alle domande facevano capire che non vi era decremento della funzione uditiva.

Anche la funzione visiva si comportò normalmente come potè constatarsi dalla registrazione su nastro magnetico magnetofonico della lettura 
delle lettere di varie grandezze di un ottotipo posto nel pannello della capsula.

Sembra che anche la visione in distanza, il senso stereoscopico e la visione dei colori siano rimasti normali.

Ton cosi la visione crepuscolare: il cosmonauta ammise che non faceva a tempo ad adattarsi al buio.

Per quanto riguarda la funzione labirintica esplorata sopratutto nel periodo di imponderabilità non vi furono segni di disturbi anche minimi.

Il cosmonauta riferisce che ogni $30^{\prime}$ durante il volo compiva una serie di movimenti della testa onde accertare se gli si fossero presentati vertigini o nausea. I movimenti consistevano nello inclinare il capo da un lato all'altro, in avanti ed all'indietro e nel muovere alternativamente le spalle.

Tali movimenti prima furono eseguiti lentamente a mano a mano che il volo procedeva sempre più rapidamente e vigorosamente fino a quanto poteva essere permesso dalla strettezza della combinazione a pressione.

Non avvertì mai alcun disturbo.

Usò anche a testa fissa il movimento laterale dei globi oculari seguendo la punta di un dito in movimento di lateralità: non ebbe difficoltà a seguire il dito senza avvertire malessere o nausea.

Riguardo alla alimentazione nulla di difficoltoso egli provò nell'ingestione degli alimenti (pasta di mele in tubo tipo dentifricio) e di g 5 di zucchero (xilosio). Poté anche mangiare senza alcuna difficoltà e deserisse il bisogno di urinare come conseguenza di un normale senso di pienezza della vescica.

L'astronauta descrisse la imponderabilità come una piacevole sensazione e asserì che la coordinazione e i movimenti muscolari si compivano normalmente.

Durante il passaggio dalla zero-gravità alle accelerazioni di rientro egli non ebbe nessun disturbo particolare, tranne che in coincidenza della accelerazione provocata dalla accensione dei retro-missili durante la quale ebbe una sensazione di moto in senso contrario al moto.

Ia stessa sensazione ma in senso inverso egli aveva de] resto avvertita durante il SECO (abbandono della capsula da parte del missile vettore) senza peraltro risentire fastidio.

Concludendo pertanto si può dire:

1. Ie risposte fisiologiche del cosmonauta Glenn durante il volo orbitale con "Amicizia 7 " alle varie situazioni del volo furono entro i 
limiti normali o entro i limiti previsti delle correnti conoscenze della medicina aeronautica.

2. Anche durante il rientro, cioè nel passaggio da zero $G$ (zero $G$ che durò sufficientemente a lungo $=4$ ore e $38^{\prime}$ ) a più $G$ non si arverò alcun chè di anormale.

Il 22 Febbraio 1961 a Colomb-Bèchar nel Sahara fu esegruito il $1^{\circ}$ esperimento spaziale francese. Venne in questa località lanciato il missile Veronique contenente il ratto "Hector".

Al ratto "Hector" furono impiantati eletrodi a livello della corteccia cerebrale, della sostanza reticolata mesencefalica, nei muscoli della nuca e nel pilastro anteriore del diaframma.

Quest'ultimo trasmetteva attraverso la stessa via, la frequenza respiratoria e la frequenza cardiaca. Tutti i dati fisiologici venivano teletrasmessi e raccolti al suolo.

L'animale era contenuto in una scatola stagna piena di $\mathrm{O}_{2}$ con sostanze assorbenti di $\mathrm{CO}_{2}$.

Durante il lancio che raggiunse i $110 \mathrm{~km}$ di quota l'animale subi una accelerazione iniziale di $9 \mathrm{G}$, e quando il missile vettore si esauri, una decelerazione di $14 \mathrm{G}$. Durante il periodo balistico l'animale venne sottoposto a rotazioni varie. Comunque mai fu sottoposto a zero-gravità.

Per quanto riguarda i dati fisiologici i risultati furono i seguenti: Durante il moto propulsivo si ebbe una diminuzione della frequenza cardiaca e respiratoria, fenomeno questo che deve imputarsi ad una reazione emozionale dell'animale (pare infatti che i ratti reacgiscono ai fatti emozionali diminuendo, anziché aumentando la loro frequenza cardiacal).

Per quanto riguarda le correnti d'azione cerebrali si notò una certa attivazione corticale e poi una tendenza alla sincronizzazione lenta del ritmo.

Il 24 Maggio 1962 fu eseguito il 20 volo orbitale americano. Tenne lanciato nello spazio il cosmonauta Malcolm Scott Carpenter, di 37 anni di età, Ufficiale della Marina Statunitense. Egli compi in meno di 5 ore, 3 giri orbitali attorno alla terra.

La capsula spaziale "Amrom 7 ", avente il peso di $1814 \mathrm{~kg}$ fu messa in orbita da un vettore Atlas (spinta $162.000 \mathrm{~kg}$ ), partendo da Cape Canaveral alle ore 7,45 locali (18 e 45 italiane) ed entrando in orbita alle ore 7,50 . La seconda orbita iniziava alle 9 e 12 e la terza alle 10 e 53. Nlle ore 12 e 19 erano accesi i razzi frenanti e alle 12 e 41 venival ragroiunta 
la superficie del mare. Alle 15 e 30 un elicottero raccoglieva l'astronauta, dopo 2 ore e 49 primi di permanenza in acqua. Il volo era durato 4 ore e 54 minuti.

Carpenter aveva eseguito il rientro agendo sui comandi manuali non avendo funzionato debitamente il sistema automatico.

Durante l'involo il cosmonauta fu sottoposto a una accelerazione di $8 \mathrm{G}$, la decelerazione in rientro ragrgiunse $7,5 \mathrm{G}$. La velocità del mezzo durante il rientro discendeva a $28.000 \mathrm{~km} / \mathrm{h}$ (velocità orbitale) a $430 \mathrm{~km} / \mathrm{h}$ in 5 minuti. Turante i periodi orbitali la capsula raggiungeva una massima distanza dalla superficie terrestre di $260 \mathrm{~km}$, mentre il perigeo era di $150 \mathrm{~km}$.

Durante i voli orbitali la temperatura dell'ambiente interno dell'abitacolo raggiunse $39 \circ \mathrm{C}\left(2^{\mathrm{a}}\right.$ orbita). La temperatura entro la tuta era di $28^{\circ}$; quella corporea di Carpenter ragoiunse $38,9{ }^{\circ} \mathrm{C}$; per questo l'astronauta ebbe una eliminazione di sudore assai intensa. La telemetrazione di grandezze fisiologiche faceva rilevare una notevole irregolarità della frequenza cardiaca, talora "incredibilmente rapida". Anche la pressione arteriosa subiva sbalzi (210/60 durante l'ultima orbita).

I)urante il volo Carpenter ha ripreso fotografie, ha constatato la possibilità di far variare l'assetto della capsula con movimenti del proprio corpo, ha osservato il comportamento di masserelle di acqua lasciate fluttuare in zero-gravità.

Il cosmonauta si alimentò con cibi pastosi in tubetti e con cibi solidi entro appositi involucri che avrebbero dovuto impedire il diffondersi di bricciole. Queste però si formarono lo stesso e poiché galleggiavano diedero parecchio fastidio al cosmonauta.

Al riguardo di questo $2^{\circ}$ volo orbitale umano si attendono però ulteriori notizie biologiche da parte dei responsabili americani.

\section{Conclustoni.}

L'esame dei dati raccolti in questa ormai consistente serie di esperimenti orbitali e suborbitali eseguiti con animali e con uomini mostra come ancora non conosciamo con chiarezza e sicurezza cosa dal punto di vista biologico accada nell'organismo lanciato nello spazio.

Da una parte vi sono elementi i quali dimostrerebbero una apparente normalità delle funzioni corporee, dall'altra vi sono elementi che ci potrebbero far supporre il contrario. Ad esempio negli esperimenti russi con gli animali traspare una certa instabilità cardio-circolatoria specialmente durante il periodo della zero-gravità, instabilità che sebbene in 
misura leggera pare sia stata riscontrata anche in Gagarin. Il prolungamento della ejezione ventricolare ed il prolungato tempo di ritorno alle condizioni normali della frequenza cardiaca, la deformazione di qualche accidente ecgrafico configurerebbero tale instabilità cardio-circolatoria.

Questa instabilità del resto era grià stata osservata da Van Bech fin dal 1953 in voli parabolici: questo autore infatti trovò il ritmo cardiaco perturbato nelle accelerazioni che seguivano il periodo di gravità, come trovó anche una noterole diminuita resistenza alle accelerazioni dopo agravità. Infatti il velo nero appariva prima del tempo e durava più a lungo.

Se questi dati venissero confermati si potrebbe supporre che vi è in regime di imponderabilità qualche cosa che turba la funzione dei barocettori, costruiti per rispondere alla accelerazione di $1 \mathrm{G}$ od anche che vi siamo perturbazioni a livello delle formazioni corticali che assiculano la regrolazione circolatoria.

Anche per quanto riguarda la funzione labirintica si notano dei contrasti sia con i cati sperimentali sia fra quanto asserito da Titov e quanto asserito dai cosmonanti americani.

Infatti i dati sperimentali (voli parabolici, Torre di subogravità) parlano per chiari disturbi labirintici estrinsecantisi sopratutto in fenomeni di incoordinazione motoria (invero migliorabili dopo allenamento) ed anche Titor descrive fenomeni di nausea e vertigini addebitabili a non regolare funzionamento del labirinto. Questi fenomeni sono stati negati dagli astronauti statunitensi e soprattutto da Glenn il quale addirittura. cercò provocarli con realizzare una fenomenologia da accelerazioni tipo Coriolis senza peraltro riuscirvi.

Anche per quanto riguarda la ingestione di alimenti vi sono pareri non uniformi: qualche autore ha infatti osservato in parecchi soggetti in istato di imponderabilità nei voli parabolici un frequente defluire attraverso il cardias verso la via esofagea delle virande ingerite quando si comprimera la parete addominale.

Come si rede dunque ancora molto ci dobbiamo aspettare sia dalle ricerche sperimentali di laboratorio, sia sopratutto dalle osservazioni di animali ed nomini lanciati in volo orbitali di più lunga durata che non quelli fino ad oggi eseguiti.

Le osservazioni certamente prenderamo altro rilievo nei futuri voli extraterrestri sia per la varietà delle componenti accelerative e di imponderabilità a cui si andrà incontro sia per la magrgiore e più prolungata sopportazione ad altra situazione che già si intravedono in questi iniziali roli orbitali. 